Revista Destaques Acadêmicos, Lajeado, v. 9, n. 3, 2017. ISSN 2176-3070

DOI: http://dx.doi.org/10.22410/issn.2176-3070.v9i3a2017.1530

www.univates.br/revistas

\title{
AVALIAÇÃO DA ATIVIDADE LEISHMANICIDA IN VITRO DE ESPÉCIES DA FAMÍLIA MYRTACEAE, NATIVAS DO SUL DO BRASIL
}

\author{
Carla Kauffmann ${ }^{1}$, Leandra Andressa Pacheco ${ }^{2}$, Bárbara Buhl ${ }^{3}$, Talita Scheibel ${ }^{4}$, \\ Elisete Maria de Freitas ${ }^{5}$, Lucélia Hoehne ${ }^{6}$, Gérzia Maria de Carvalho Machado ${ }^{7}$, \\ Marilene Marcuzzo do Canto Cavalheiro ${ }^{8}$, Simone Cristina Baggio Gnoatto ${ }^{9}$, \\ Eduardo Miranda Ethur ${ }^{10}$
}

Resumo: Leishmaniose é uma doença causada por protozoários do gênero Leishmania, sendo esses transmitidos por um inseto vetor, mosquito palha, e que pode apresentar como hospedeiro animais silvestres ou domésticos e o homem. A apresentação clínica da doença pode variar de cutânea, mucocutânea, cutânea difusa a visceral. Considerando

1 Farmacêutica. Mestre em Ciências Farmacêuticas. Doutora em Ambiente e Desenvolvimento. Professora do Centro de Ciências Biológicas e da Saúde, Univates. E-mail: carlakauffmann@univates.br

2 Biomédica. Mestranda no Programa de Pós-Graduação em Biotecnologia, Univates. E-mail: leandrapacheco@universo.univates.br

3 Acadêmica do Curso de Farmácia, Univates. E-mail: barbara.buhl@univates.br

4 Acadêmica do Curso de Farmácia, Univates. E-mail: talisc95@gmail.com

5 Programas de Pós-graduação em Biotecnologia e Ensino, Laboratório de Propagação de Plantas. E-mail: elicauf@univates.br

6 Doutora em Química, Programa de Pós-Graduação em Biotecnologia, Univates. E-mail: luceliah@univates.br

7 Pesquisadora. Laboratório de Bioquímica de Tripanossomatídeos, Instituto Oswaldo Cruz (FIOCRUZ). E-mail: machado@ioc.fiocruz.br

8 Doutora em Química. Pesquisadora. Laboratório de Bioquímica de Tripanossomatídeos, Instituto Oswaldo Cruz (FIOCRUZ). E-mail: mcantoca@ioc.fiocruz.br

9 Farmacêutica. Doutora em Ciências Farmacêuticas. Professora do Curso de Farmácia, UFRGS. E-mail: simone.gnoatto@ufrgs.br

10 Doutor em Química.Professor do Programa dePós-Graduaçãoem Ambientee Desenvolvimento (PPGAD), do Programa de Pós-Graduação em Biotecnologia e do Centro de Ciências Exatas e Tecnológicas, Univates. E-mail: eduardome@univates.br 
a diversidade da flora nacional e seu potencial biológico, assim como o fato que nosso país constitui-se em área endêmica de leishmanioses, o presente trabalho objetivou analisar as espécies Calyptranthes tricona, Eugenia anomala, Eugenia pitanga, Eugenia pyriformis, Myrciaria plinioides, Myrrhinium atropurpureum e Psidium salutare quanto à sua atividade leishmanicida in vitro contra formas promastigotas de Leishmania amazonensis. Extratos aquosos e hidroalcóolicos foram preparados através de decocção e maceração, respectivamente. A atividade leishmanicida dos extratos foi analisada in vitro contra formas promastigotas de L. amazonensis empregando-se adaptação do ensaio de MTT. Os extratos hidroalcoólico e aquoso de M. plinioides, extrato hidroalcóolico de E. pyriformis e extrato aquoso de $P$. salutare apresentaram atividade significativa, comparável ao fármaco padrão, pentamidina. Dessa forma, tem-se como perspectiva a continuidade dos estudos com essas espécies a fim de elucidar constituição química e outros aspectos relativos à atividade biológica.

Palavras-chave: Atividade leishmanicida. Leishmania amazonenses. Myrtaceae. Bioma Pampa. Bioma Mata Atlântica.

\section{INTRODUÇÃO}

Leishmaniose é uma doença infecciosa causada por protozoários pertencentes ao gênero Leishmania, que pode se apresentar em diferentes apresentações clínicas: leishmaniose visceral (LV), leishmaniose cutânea (LC), leishmaniose mucocutânea (LMC) e leishmaniose cutânea difusa (LCD). A doença é considerada uma antropozoonose por apresentar um hospedeiro invertebrado, flebotomíneos, conhecido popularmente como mosquito palha, o qual é responsável pela transmissão das formas promastigotas a animais silvestres e domésticos, assim como a humanos. No hospedeiro vertebrado, a forma promastigota é endocitada pelos macrófagos, onde se transforma na forma amastigota e desta forma propaga a infecção (REY, 2002; MICHALICK, 2003; CAMARGO, 2008; BRASIL, 2014).

As leishmanioses geralmente afetam populações de regiões tropicais e subtropicais, com baixo poder econômico e que residem nas áreas periurbanas. A transmissão da doença, que se restringia a áreas próximas a florestas, tem se ampliado, fato relacionado a mudanças ambientais, climáticas e econômicas, entre outras. A dificuldade no tratamento de leishmanioses relaciona-se a inexistência de vacinas para humanos, assim como a eficácia relativa e toxicidade dos fármacos existentes. Pelas características listadas, leishmaniose é considerada uma Doença Negligenciada Tropical (DNT) pela Organização Mundial da Saúde (OMS) (GARCIA et al., 2011; BOECHAT; MAGALHÃES, 2012; DIAS et al., 2013; WHO, 2015; GILLESPIE et al., 2016; WHO, 2016).

Segundo a OMS, anualmente, 300.000 novos casos de LV e um milhão de novos casos de LC ou LMC são registrados, o que totaliza aproximadamente 1,3 milhões de pessoas atingidas pela doença. Soma-se, ainda, 50.000 mortes que podem ser causadas, anualmente, por leishmanioses. O Brasil é uma área endêmica de leishmanioses - visceral, cutânea e mucocutânea. Entre 2016 e o início de 2017, três casos de óbitos por LV foram registrados no Rio Grande do 
Sul, no município de Porto Alegre, além de outros dois casos que evoluíram para cura com o tratamento (WHO, 2015; WHO, 2016; RIO GRANDE DO SUL, 2017).

No Brasil, a forma visceral da doença é causada por Leishmania donovani, enquanto que as formas cutânea e mucocutânea são provocadas pela infecção com Leishmania tropica, Leishmania braziliensis e Leishmania amazonensis (CAMARGO, 2008). Considerando o impacto sanitário de leishmanioses e a possibilidade de desenvolvimento de novos medicamentos a partir de fontes naturais, como plantas, o presente estudo objetivou avaliar a atividade leishmanicida in vitro contra formas promastigotas de L. amazonensis de extratos hidroalcoólicos e aquosos de espécies pertencentes à família Myrtaceae, nativas do Rio Grande do Sul.

A família Myrtaceae encontra-se distribuída entre diferentes biomas brasileiros, como a Floresta Atlântica, o Pampa, a Restinga e o Cerrado, sendo uma das principais famílias da flora de nosso país (SOUZA; LORENZI, 2012). Espécies pertencentes à família são empregadas popularmente com diferentes finalidades, como por exemplo, no tratamento de diarreia, asma e inflamações (Myrciaria cauliflora, jabuticabeira); no tratamento de malária (Myrciaria dubia); no tratamento de diarreias, febre e reumatismo (Eugenia uniflora, pitangueira) (CORRÊA, 1984; SIMÕES et al., 1989; LORENZI; MATOS, 2002; BOSCOLO; VALLE, 2008; RUIZ et al., 2011). Contudo, estudos sobre atividade biológica de espécies nativas dos biomas Mata Atlântica e Pampa, como Calyptranthes tricona, Eugenia anomala, Eugenia arenosa, Eugenia pyriformis, Myrciaria plinioides, Myrrhinium atropurpureum e Psidium salutare, são escassos, sendo essas alvo do presente trabalho.

\section{MATERIAL E MÉTODOS}

\subsection{Material vegetal}

O material vegetal, constituído de folhas de espécies nativas da família Myrtaceae, foi coletado em diferentes municípios do Rio Grande do Sul, sendo sua identificação realizada pela Professora Dra. Elisete Maria de Freitas, botânica da Universidade do Vale do Taquari - Univates. Exsicata de cada espécie foi depositada no Herbário do Vale do Taquari (HVAT), do Museu de Ciências Naturais da Univates (Quadro 1). 
Quadro 1. Espécies da família Myrtaceae, nativas do sul do Brasil, empregadas no estudo

\begin{tabular}{|c|c|c|c|}
\hline Espécie & Nome popular & $\begin{array}{l}\text { Local de coleta/ } \\
\text { Bioma }\end{array}$ & Exsicata \\
\hline $\begin{array}{l}\text { Calyptranthes tricona } \\
\text { D.Legrand }\end{array}$ & guaburiti $^{\text {a }}$ & $\begin{array}{l}\text { Lajeado } \\
\text { Mata Atlântica }\end{array}$ & HVAT 4496 \\
\hline $\begin{array}{l}\text { Eugenia anomala } \\
\text { D.Legrand }\end{array}$ & ------ & $\begin{array}{l}\text { Alegrete } \\
\text { Pampa }\end{array}$ & HVAT 4058 \\
\hline $\begin{array}{l}\text { Eugenia pitanga (O.Berg) } \\
\text { Nied. }\end{array}$ & pitanga-do-campo $^{\text {b }}$ & $\begin{array}{l}\text { Alegrete } \\
\text { Pampa }\end{array}$ & HVAT 4052 \\
\hline $\begin{array}{l}\text { Eugenia pyriformis } \\
\text { Cambess. }\end{array}$ & uvaia $^{a}$ & $\begin{array}{l}\text { Cruzeiro do Sul } \\
\text { Mata Atlântica }\end{array}$ & HVAT 3833 \\
\hline $\begin{array}{l}\text { Myrciaria plinioides } \\
\text { D.Legrand }\end{array}$ & $\begin{array}{l}\text { camboim, cambuim } \\
\text { or cambuía }^{\text {a }}\end{array}$ & $\begin{array}{l}\text { Lajeado } \\
\text { Mata Atlântica }\end{array}$ & HVAT 1066 \\
\hline $\begin{array}{l}\text { Myrrhinium atropurpureum } \\
\text { Schott }\end{array}$ & carrapato, pau-ferro ${ }^{a}$ & $\begin{array}{l}\text { Canudos do Vale } \\
\text { Mata Atlântica }\end{array}$ & HVAT 2718 \\
\hline $\begin{array}{l}\text { Psidium salutare var. } \\
\text { sericeum (Cambess.) } \\
\text { Landrum }\end{array}$ & araçá-do-campo ${ }^{\mathrm{b}}$ & $\begin{array}{l}\text { Alegrete } \\
\text { Pampa }\end{array}$ & HVAT 4051 \\
\hline
\end{tabular}

a Sobral et al., 2013.

${ }^{\mathrm{b}}$ Flora Digital, 2010.

\subsection{Preparação dos extratos}

As folhas das espécies analisadas foram secas em temperatura ambiente e, após, trituradas em liquidificador industrial (Metvisa $\left.{ }^{\circledR}\right)$. Amostras das espécies foram submetidas ao processo de maceração com etanol 90\%, na proporção 1:10 de droga:solvente $(\mathrm{m} / \mathrm{v})$, por 7 dias, obtendo-se, desta forma, os extratos hidroalcoólicos. Na preparação de extratos aquosos, o material vegetal foi misturado com água fervente, na proporção 1:10 de droga:solvente $(\mathrm{m} / \mathrm{v})$, e subsequente fervido por 15 minutos. Após a realização de cada processo extrativo, o produto resultante foi filtrado e concentrado em evaporador rotatório sob pressão reduzida (Rotavapor ${ }^{\circledR} \mathrm{R}-100$, Büchi Labortechnik), em temperatura inferior a $50^{\circ} \mathrm{C}$, para eliminação total do solvente. 


\subsection{Avaliação da atividade leishmanicida in vitro contra formas promastigotas de Leishmania amazonensis}

\subsubsection{Amostras}

$\mathrm{Na}$ avaliação da atividade leishmanicida in vitro foram empregadas alíquotas dos extratos hidroalcoólicos e dos extratos aquosos das espécies $C$. tricona, E. anomala, E. arenosa, E. pyriformis, M. plinioides, M. atropurpureum e $P$. salutare.

\subsubsection{Parasitos}

Promatisgotas de L. amazonensis (cepa MHOM/BR/77/LTB0016) foram cultivados em meio Schneider, $\mathrm{pH} 7,2$, suplementado com 10\% (v/v) de soro fetal bovino (SFB), mantidos em estufa de demanda bioquímica de oxigênio (BOD) a $26^{\circ} \mathrm{C}$. Os parasitas foram coletados no quarto dia de cultivo, quando a porcentagem de formas infectantes (promastigotas metacíclicas) é maior, contados em câmara de Neubauer e ajustados a uma concentração de 1x10 parasitas/mL, usando o sobrenadante como diluente.

\subsubsection{Ensaio in vitro de avaliação da atividade leishmanicida}

Alíquotas das amostras ou de isetionato de pentamidina (Sideron ${ }^{\circledR}$, Ítaca Laboratórios), fármaco empregado como padrão, foram dissolvidos em solução aquosa de dimetilsulfóxido (DMSO) $(10 \mathrm{mg} / \mathrm{mL})$. A concentração de DMSO nas soluções empregadas no ensaio não ultrapassou $1,4 \%$ (v/v), concentração máxima em que o reagente não provoca toxicidade para os parasitos (CANTOCAVALHEIRO et al., 1997).

O ensaio foi realizado em placas de 96 poços de fundo chato (Costar, Corning Incorporated). A cada poço foi adicionado $100 \mu \mathrm{L}$ da suspensão de promastigotas, na concentração de $1 \times 10^{7}$ parasitas $/ \mathrm{mL}$. Alíquotas das amostras ou do padrão foram adicionadas à primeira linha de poços, realizando-se diluição seriada. As amostras foram testadas em concentrações que variaram de 0,156 a $80 \mu \mathrm{g} / \mathrm{mL}$. As placas foram incubadas, em estufa BOD, por 24 horas, a $26^{\circ} \mathrm{C}$.

Os promastigotas viáveis foram avaliados empregando uma versão modificada do ensaio com brometo de 3-(4,5-dimetiltiazol-2-il)-2,5-difeniltetrazolio (MTT) (DUTTA et al., 2005). Alíquota de $22 \mu \mathrm{L}$ de solução de MTT $0,5 \%$ foi adicionada a cada poço e a placa foi incubada, ao abrigo da luz, por 4 horas. Após, $80 \mu \mathrm{L}$ de DMSO foi adicionado a cada poço e a absorbância foi determinada em $570 \mathrm{~nm}$, empregando-se espectrofotômetro de microplacas $\left(\mu Q u a n t^{\mathrm{TM}}\right.$, BioTek). A percentagem média de promastigotas viáveis foi calculada a partir da fórmula: 
Os valores de $\mathrm{IC}_{50}$ para as amostras e padrão, isto é, a concentração que inibe o crescimento dos parasitos em $50 \%$, foram determinados.

\subsubsection{Análise estatística da atividade leishmanicida}

$\mathrm{O}$ ensaio foi realizado em triplicata e em três experimentos independentes. Os valores de $\mathrm{IC}_{50}$ foram determinados por análise de regressão logarítmica, empregando o software GraphPad Prism 5.0, sendo os resultados da atividade leishmanicida in vitro expressos como média \pm desvio-padrão. Os resultados obtidos foram analisados empregando-se teste $t$ de Student, empregando o software BioEstat 5.0, considerando-se valor de $p \leq 0,05$ como diferença significativa.

\section{RESULTADOS E DISCUSSÃO}

No Brasil, L. amazonensis causa ulcerações na pele e nas mucosas, estando associada com diferentes formas clínicas de leishmaniose, incluindo cutânea, mucocutânea, cutânea difusa e visceral (LEON et al., 1990; WHO, 2010). A realização de ensaios para avaliação de atividade leishmanicida com formas promastigotas apresenta vantagens em comparação com o uso de formas amastigotas, como facilidade de execução e menor custo, sendo uma estratégia de screening na busca de novas moléculas bioativas (SIQUEIRA-NETO et al., 2010).

Extratos aquosos e hidroalcoólicos de folhas de espécies da família Myrtaceae tiveram seu potencial leishmanicida in vitro avaliado contra formas promastigotas de L. amazonensis. Isetionato de pentamidina foi utilizado como fármaco padrão no ensaio e apresentou valor de $\mathrm{IC}_{50}$ de $23,22 \pm 9,04 \mu \mathrm{g} / \mathrm{mL}$. Os extratos hidroalcoólico e aquoso de M. plinioides, assim como o extrato hidroalcóolico de E. pyriformis e o extrato aquoso de $P$. salutare, foram tão ativos quanto à pentamidina contra promastigotas de L. amazonensis, apresentando valores de $\mathrm{IC}_{50}$ de $8,77 \pm 3,77 \mu \mathrm{g} / \mathrm{mL}, 9,21 \pm 3,31 \mu \mathrm{g} / \mathrm{mL}, 14,42 \pm 7,48 \mu \mathrm{g} / \mathrm{mL}$ e $37,03 \pm 8,01 \mu g / m L$, respectivamente (TABELA 1 ). 
Tabela 1. Valores de $\mathrm{IC}_{50}(\mu \mathrm{g} / \mathrm{mL})$ de extratos hidroalcoólicos e aquosos de folhas de espécies da família Myrtaceae, nativas do sul do Brasil, contra formas promastigotas de Leishmania amazonensis

\section{Espécie/Extrato}

$$
\mathrm{IC}_{50}(\mu \mathrm{g} / \mathrm{mL})
$$

\section{L. amazonensis}

\section{Calyptranthes tricona}

Extrato hidroalcoólico

Extrato aquoso

\section{Eugenia anomala}

Extrato hidroalcoólico

Extrato aquoso

\section{Eugenia pitanga}

Extrato hidroalcoólico

Extrato aquoso

\section{Eugenia pyriformis}

Extrato hidroalcoólico

Extrato aquoso

Myrciaria plinioides

Extrato hidroalcoólico

Extrato aquoso

Myrrhinium atropurpureum

Extrato hidroalcoólico

Extrato aquoso

\section{Psidium salutare}

Extrato hidroalcoólico

Extrato aquoso

Isetionato de pentamidina ${ }^{a}$
$>80 \mu \mathrm{g} / \mathrm{mL}$

$>80 \mu \mathrm{g} / \mathrm{mL}$

$>80 \mu \mathrm{g} / \mathrm{mL}$

$>80 \mu \mathrm{g} / \mathrm{mL}$

$48,51 \pm 2,02^{*}$

$>80 \mu \mathrm{g} / \mathrm{mL}$

$14,42 \pm 7,48$

$50,41 \pm 5,26^{*}$

$8,77 \pm 3,77$

$9,21 \pm 3,31$

$45,24 \pm 5,90^{*}$

$>80 \mu \mathrm{g} / \mathrm{mL}$

$59,37 \pm 5,97^{*}$

$37,03 \pm 8,01$

$23,22 \pm 9,04$

aFármaco padrão

*Valores são estatisticamente diferentes do fármaco padrão $(p \leq 0.05)$.

Resultados expressos como média dos valores \pm desvio-padrão

Os extratos hidroalcoólicos de E. pitanga, M. atropurpureum e P. salutare, assim como o extrato aquoso de E. pyriformis, demonstraram moderada atividade contra $L$. amazonensis, com valores de $\mathrm{IC}_{50}$ de 48,51 $\pm 2,02 \mu \mathrm{g} / \mathrm{mL}$, e $45,24 \pm 5,90 \mu \mathrm{g} / \mathrm{mL}, 59.37 \pm 5.97 \mu \mathrm{g} / \mathrm{mL}$ e 50,41 $\pm 5,26 \mu \mathrm{g} / \mathrm{mL}$, respectivamente. Entretanto, os extratos aquoso e hidroalcóolico de C. tricona e E. anomala, assim como os extratos aquosos de E. pitanga e $M$. atropurpureum, foram inativos, com valores de $\mathrm{IC}_{50}$ superiores a $80 \mu \mathrm{g} / \mathrm{mL}$ (TABELA 1). 
Espécies pertencentes à família Myrtaceae têm sido avaliadas quanto a sua potencial atividade leishmanicida. Dessa forma, ação leishmanicida contra formas amastigotas de L. amazonensis foi observada para frações isoladas a partir do extrato folhas de Blepharocalyx salicifolius, em concentrações que variaram de 19 a $29 \mathrm{\mu g} / \mathrm{mL}$ (SIQUEIRA et al., 2010). Estudos realizados com extratos de Campomanesia eugenioides, de Myrcia linearifolia e de Psidium guajava, não demonstraram ação in vitro considerável contra formas promastigotas de L. amazonensis, com valores de $\mathrm{IC}_{50}$ superiores a $100 \mu \mathrm{g} / \mathrm{mL}$ (LUIZE et al., 2005; MOURA-COSTA et al., 2012; COSTA et al., 2014).

Enquanto que estudos realizados com espécies do gênero Eugenia demonstraram o potencial do extrato hexânico de frutos de E. umbelliflora contra formas promastigotas de L. amazonensis e L. brasiliensis, com valores de $\mathrm{IC}_{50}$ de $14,3 \mu \mathrm{g} / \mathrm{mL}$ e $5,7 \mu \mathrm{g} / \mathrm{mL}$, respectivamente (CECHINEL FILHO et al., 2013). Contudo para extratos hexânico e metanólico de folhas E. uniflora não se verificou atividade promissora contra formas promastigotas de $L$. amazonensis, apresentando valores de $\mathrm{IC}_{50}$ maiores que $200 \mu \mathrm{g} / \mathrm{mL}$ e $250 \mu \mathrm{g}$ / $\mathrm{mL}$, respectivamente (BRAGA et al., 2007; RIBEIRO et al., 2014).

O emprego de plantas tem desempenhado papel de destaque no desenvolvimento de fármacos e o uso de abordagem multidisciplinar, combinando, por exemplo, metodologias de síntese combinatória e manipulação de vias biossintéticas, poderão otimizar a cadeia produtiva (SCHENKEL; GOSMANN; PETROVICK, 1999; HOSTETTMANN et al., 2008; NEWMAN; CRAGG, 2016). A partir do presente estudo foi possível identificar o potencial das espécies E. pyriformis, M. plinioides e $P$. salutare, as quais apresentaram atividade comparável ao fármaco padrão, pentamidina, contra formas promastigotas de L. amazonensis.

No Brasil são encontrados seis biomas: Amazônia, Mata Atlântica, Caatinga, Cerrado, Pantanal e Pampa. O bioma Pampa localiza-se exclusivamente na metade sul do Rio Grande do Sul, ocupando 63\% do território do Estado, sendo o restante ocupado pela Mata Atlântica (IBGE, 2004). O bioma Pampa apresenta vegetação diversificada e adaptada ao clima, ao solo e ao manejo, contudo esta tem sofrido modificações em função da expansão da pecuária, da agricultura e da silvicultura, bem como da urbanização. Assim, $54 \%$ da área deste bioma não apresenta mais vegetação original, enquanto que apenas 7,48\% do bioma Mata Atlântica é remanescente no Estado (BOLDRINI, 2009; BRASIL, 2011; OVERBECK et al., 2013).

Espécies nativas do bioma Pampa, como E. anomala, encontram-se em perigo de extinção (RIO GRANDE DO SUL, 2014). A presença de E. anomala e E. pitanga foi relatada em áreas de areais no bioma Pampa, sendo essas consideradas espécies mais resistentes por apresentarem raízes profundas e folhas coriáceas (FREITAS et al., 2010). O crescimento dessas espécies em condições adversas pode favorecer a produção de metabólitos secundários distintos ou em maior concentração a fim de torná-las mais resistentes ao meio, tornando-as atrativas 
como possíveis fontes de moléculas bioativas, ainda o risco de extinção das mesmas denota a importância de estudos interdisciplinares.

A perspectiva de uso de espécies nativas como possível fonte para o desenvolvimento de medicamentos reforça a necessidade e relevância de estudos sobre a manutenção dessas espécies, prioritariamente daquelas em perigo de extinção, como E. anomala (RIO GRANDE DO SUL, 2014). Valls et al. (2009) expõem a preocupação com a exploração de espécies nativas e sugerem a criação de programas de adaptação ao cultivo, assim como o desenvolvimento de conhecimento científico sobre a variabilidade genética destas, a fim de evitar o extrativismo.

\section{CONCLUSÃO}

Os resultados observados para os extratos hidroalcoólico e aquoso de $M$. plinioides, extrato hidroalcóolico de E. pyriformis e extrato aquoso de $P$. salutare são promissores, uma vez que esses apresentaram ação leishmanicida comparável à pentamidina. Além disso, a ação demonstrada por estes extratos destaca-se entre aqueles apresentados por outras espécies pertencentes à família Myrtaceae. Assim, vislumbra-se a perspectiva de continuidade dos estudos com essas espécies, englobando a constituição química dos extratos, a avaliação da atividade leishmanicida contra formas amastigotas e a citotoxicidade em macrófagos, além de elucidação de possíveis mecanismos envolvidos na atividade biológica.

\section{AGRADECIMENTOS}

Este estudo recebeu apoio financeiro da Fundação de Amparo à Pesquisa do Estado do Rio Grande do Sul (FAPERGS) e do Conselho Nacional de Desenvolvimento Científico e Tecnológico (CNPq; PRONEX 10/0029-0).

\section{REFERÊNCIAS}

BOECHAT, N.; MAGALHÃES, J. Era uma vez... Doenças Negligenciadas. Revista Virtual de Química, v. 4, n. 3, p. 195-196, 2012.

BOLDRINI, I. I. A flora dos campos do Rio Grande do Sul. In: PILLAR, V. P.; MÜLLER, S. C.; CASTILHOS, Z. M. S.; JACQUES, A. V. Á. Campos Sulinos conservação e uso sustentável da biodiversidade. Brasília: MMA, 2009. p. 63-77.

BOSCOLO, O. H.; VALLE, L.S. Plantas de uso medicinal em Quissamã, Rio de Janeiro, Brasil. Iheringia, v. 63, n. 2, p. 263-277, 2008.

BRAGA, F. G.; BOUZADA, M. L. M.; FABRI, R. L.; MATOS, M. O.; MOREIRA, F. O.; SCIO, E.; COIMBRA, E. S. Antileishmanial and antifungal activity of plants used in traditional medicine in Brazil. Journal of Ethnopharmacology, v. 111, n. 2, p. 396-402, 2007. 
BRASIL. Ministério da Ciência e Tecnologia. Instituto Nacional de Pesquisas Espaciais - INPE. Fundação SOS Mata Atlântica. Atlas dos remanescentes florestais da Mata Atlântica período 2008-2010. São José dos Campos, SP: INPE, 2011. 120 p.

BRASIL. Ministério da Saúde. Secretaria de Vigilância em Saúde. Departamento de Vigilância Epidemiológica. Manual de vigilância e controle da leishmaniose visceral. 1. ed., 5. reimpr. Brasília: Editora do Ministério da Saúde, 2014. 120 p.

CAMARGO, E. P. Doenças tropicais. Estudos Avançados, v. 22, n. 64, p. 95-100, 2008.

CANTO-CAVALHEIRO, M. M.; ESCHEVARRIA, A.; ARAUJO, C. A.; BRAVO, M. F.; SANTOS, L. H. S.; JANSEN, A. M.; LEON, L. L. The potencial effects of new synthetic drugs against Leishmania amazonensis and Trypanosoma cruzi. Microbios, v. 90, n. 362, p. 51-60, 1997.

CECHINEL FILHO, V.; MEYRE-SILVA, C.; NIERO, R.; MARIANO, N. L. B.; NASCIMENTO, F. G.; FARIAS, I. V.; GAZONI, V. F.; SILVA, B. S.; GIMÉNEZ, A.; GUTIERREZ-YAPU, D.; SALAMANCA, E.; MALHEIROS, A. Evaluation of antileishmanial activity of selected brazilian plants and identification of the active principles. Evidence-Based Complementary and Alternative Medicine, v. 2013, 7 p., 2013. Article ID 265025. Disponível em: <http:/ / dx.doi. org/10.1155/2013/2650252013>. Acesso em: 25 ago. 2016.

CORREAA, P. Dicionário das Plantas Úteis do Brasil e das Exóticas Cultivadas, vol. 5. Rio de Janeiro: Imceprensa Nacional, 1984. p. 508-512.

COSTA, R. C.; SANTANA, D. B.; ARAÚJO, R. M.; PAULA, J. E.; NASCIMENTO, P. C.; LOPES, N. P.; BRAZ-FILHO, R.; ESPINDOLA, L. S. Discovery of the rapanone and suberonone mixture as a motif for leishmanicidal and antifungal applications. Bioorganic \& Medicinal Chemistry, v. 22, n. 1, p. 135-140, 2014.

DIAS, L. C.; DESSOY, M. A.; GUIDO, R. V. C.; OLIVA, G.; ANDRICOPULO, A. D. Doenças tropicais negligenciadas: uma nova era de desafios e oportunidades. Química Nova, v. 36, n. 10, p. 1552-1556, 2013 b.

DUTTA, A.; BANDYOPADHYAY, S.; MANDAL, C.; CHATTERJEE, M. Development of a modified MTT assay for screening antimonial resistant field isolates of Indian visceral leishmaniasis. Parasitology International, v. 54, p. 119-122, 2005.

FLORA DIGITAL DO RIO GRANDE DO SUL E DE SANTA CATARINA: Eugenia pitanga (O.Berg) Nied. Disponível em: <http:/ / www.ufrgs.br/fitoecologia/florars / index.php?pag=buscar_mini.php\&especie=2390 >. Acesso em: 12 set. 2016.

FLORA DIGITAL DO RIO GRANDE DO SUL E DE SANTA CATARINA: Myrciaria plinioides. Disponível em: <http://www.ufrgs.br/fitoecologia/florars/open_ sp.php?img=272>. Acesso em: 12 set. 2016. 
FLORA DIGITAL DO RIO GRANDE DO SUL E DE SANTA CATARINA: Psidium salutare. Disponível em: <http:/ / www.ufrgs.br/fitoecologia/florars/open_ sp.php?img=10877>. Acesso em: 12 set. 2016.

FREITAS, E. M.; TREVISAN, R.; SCHNEIDER, A. A.; BOLDRINI, I. I.Floristic diversity in areas of sandy soil grasslands in Southwestern Rio Grande do Sul, Brazil. Revista brasileira de Biociências, v. 8, n. 1, p. 112-130, 2010.

GARCIA, L. P.; DE MAGALHÃES, L. C. G.; AUREA, A. P.; DOS SANTOS, C. F.; DE ALMEIDA, R. F. Epidemiologia das doenças negligenciadas no Brasil e gastos federais com medicamentos - Texto para Discussão. Brasília, DF: Instituto de Pesquisa Econômica Aplicada (IPEA), n. 1607, 2011.

GILLESPIE, P. M.; BEAUMIER, C. M.; STRYCH, U.; HAYWARD, T.; HOTEZ, P. J.; BOTTAZZI; M. E. Status of vaccine research and development of vaccines for leishmaniasis. Vaccine, v. 34, n. 26, p. 2992-2995, 2016.

HOSTETTMANN, K.; GUPTA, M. P.; MARSTON, A.; QUEIROZ, E. F. Handbook of strategies for the isolation of bioactive natural products. Bogota: Iberoamerican program of Science and technology, 2008. p.13-14.

INSTITUTO BRASILEIRO DE GEOGRAFIA E ESTATÍSTICA - IBGE 2004. Mapa da vegetação do Brasil e Mapa de Biomas do Brasil. Disponível em: <http:/ / www.ibge. gov.br/home/presidencia/noticias/21052004biomashtml.shtm>. Acesso em: 12 set. 2016.

LEON, L. L.; MACHADO, G. M.; PAES, L. E.; GRIMALDI JR., G. Antigenic variation of Leishmania amazonensis isolates causing diffuse cutaneous leishmaniasis (DCL).

Transactions of the Royal Society of Tropical Medicine \& Hygiene, v. 84, p. 678-680, 1990.

LORENZI, Harri; MATOS, Francisco José de Abreu. Plantas medicinais no Brasil: nativas e exóticas. Nova Odessa: Instituto Plantarum, 2002. p. 350-351.

LUIZE, P. S.; TIUMAN, T. S.; MORELLO, L. G.; MAZA, P. K.; UEDA-NAKAMURA, T.; DIAS FILHO, B. P.; CORTEZ, D. A. G.; MELLO, J. C. P. M.; NAKAMURA, C. V. Effects of medicinal plant extracts on growth of Leishmania (L.) amazonensis and Trypanosoma cruzi. Revista Brasileira de Ciências Farmacêuticas, v. 41, n.1, p. 85-94, 2005.

MICHALICK, M. S. M. Gênero Leishmania. In: NEVES, David Pereira. Parasitologia humana. 10. ed. São Paulo: Atheneu, 2003. p. 31-35.

MOURA-COSTA, G. F.; NOCCHI, S. R.; CEOLE, L. F.; MELLO, J. C. P.; NAKAMURA, C. V.; DIAS FILHO, B. P.; TEMPONI, L. G.; UEDA-NAKAMURA, T. Antimicrobial activity of plants used as medicinals on an indigenous reserve in Rio das Cobras, Paraná, Brazil. Journal of Ethnopharmacology, v. 143, n. 2, p. 631-638, 2012. 
NEWMAN, D. J.; CRAGG, G. M. Natural products as sources of new drugs from 1981 to 2014. Journal of Natural Products, v. 79, p. 629-661, 2016.

OVERBECK, G. E.; HERMANN, J. M.; ANDRADE, B. O.; BOLDRINI, I. I.; KIEHL, K.; KIRMER, A.; KOCH, C.; KOLLMANN, J.; MEYER, S. T.; MÜLLER, S. C.; NABINGER, C.; PILGER, G. E.; TRINDADE, J. P. P.; VÉLEZ-MARTIN, E.; WALKER, E. A.; ZIMMERMANN, D. G.; PILLAR, V. D. Restoration Ecology in Brazil - Time to Step Out of the Forest. Natureza \& Conservação, v. 11, n. 1, p. 92-95, 2013.

REY, L. Bases da parasitologia medica. 2. ed. Rio de Janeiro: Guanabara Koogan, 2002. p. 50-70.

RIBEIRO, T. G.; CHÁVEZ-FUMAGALLI, M. G.; VALADARES, D. G.; FRANCA, J. R.; LAGE, P. S.; DUARTE, M. C.; ANDRADE, P. H. R.; MARTINS, V. T.; COSTA, L. E.; ARRUDA, A. L. A.; FARACO, A. A. G.; COELHO, E. A. F.; CASTILHO, R. O. Antileishmanial activity and cytotoxicity of Brazilian plants. Experimental Parasitology, v. 143, p. 60-68, 2014.

RIO GRANDE DO SUL. Decreto Lei n 52109 de 14 de dezembro de 2014. Declara as espécies da flora native ameaçadas de extinção no Estado do Rio Grande do Sul. Porto Alegre: Diário Oficial do Estado, 02 de dezembro de 2014, p. 2 - 3.

RIO GRANDE DO SUL. Secretaria Estadual da Saúde. Disponível em: <http:/ /www. cevs.rs.gov.br/upload/arquivos/201705/08103840-apresentacao-leishmaniosevisceral-humana.pdf>. Acesso em: 21 ago. 2017.

RUIZ, L.; RUIZ, L.; MACO, M.; COBOS, M.; GUTIERREZ-CHOQUEVILCA, A. L.; ROUMY, V. Plants used by native Amazonian groups from the Nanay River (Peru) for the treatment of malaria. Journal of Ethnopharmacology, v. 133, n. 2, p. 917-921, 2011.

SCHENKEL, E. P.; GOSMANN, G.; PETROVICK, P. R. Produtos de origem vegetal e o desenvolvimento de medicamentos. In: SIMÕES, C. M. O.; SCHENKEL, E. P.; GOSMANN, G.; MELLO, J. C. P.; MENTZ, L. A.; PETROVICK, P. R. (Org.). Farmacognosia: da planta ao medicamento. Porto Alegre/Florianópolis: Editora da UFRGS/Editora da UFSC, 1999. p. 291-320.

SIMÕES, C. M. O.; MENTZ, L. A.; SCHENKEL, E. P.; IRGANG, B. E.; STEHMANN, J. R. Plantas da Medicina Popular no Rio Grande do Sul. 3. ed. Porto Alegre: Editora da Universidade/UFRGS, 1989. 174p.

SIQUEIRA, E. P.; SOUZA-FAGUNDES, E. M.; SOBRAL, M. E. G.; ALVES, T. M. A.; RABELLO, A.; ZANI, C. L. Leishmanicidal activities of the extract from Blepharocalyx salicifolius (Kunth) O. Berg, Myrtaceae. Revista Brasileira de Farmacognosia, v. 20, n. 3, p. 416-421, 2010.

SIQUEIRA-NETO, J. L.; SONG, O.; OH, H.; SOHN, J.; YANG, G.; NAM, J.; JANG, J.; CECHETTO, J.; LEE, C. B.; MOON, S.; GENOVESIO, A.; CHATELAIN, E.; CHRISTOPHE, T.; FREITAS-JÚNIOR, L.H. Antileishmanial high-throughput drug 
screening reveals drug candidates with new scaffolds. PLoS Neglected Tropical Disease, v. 4, n. 5, p. e675, 2010.

SOBRAL, M.; JARENKOW, J. A.; BRACK, P.; IRGANG, B.; LAROCCA, J.; RODRIGUES, R. S. Flora Arbórea e Arborescente do Rio Grande do Sul, Brasil. 2 ed. São Carlos: Rima, 2013. p. 130-131.

SOUZA, V. C.; LORENZI, H. Botânica sistemática: guia ilustrado para identificação das famílias de fanerógamas nativas e exóticas no Brasil, baseado em APG III. 3. ed. Nova Odessa: Instituto Plantarum, 2012. p. 428-429.

VALLS, J. F. M.; BOLDRINI, I. I.; LONGHI-WAGNER, H. M.; MIOTTO, S. T. S. O patrimônio florístico dos Campos: potencialidades de uso e a conservação de seus recursos genéticos. In: PILLAR, V. P.; MÜLLER, S. C.; CASTILHOS, Z. M. S.; JACQUES, A. V. Á. Campos Sulinos - conservação e uso sustentável da biodiversidade (Editores). Brasília: MMA, 2009. p. 139-154.

WORLD HEALTH ORGANIZATION - WHO. Control of the leishmaniasis: report of a meeting of the WHO Expert Committee on the Control of Leishmaniases, Geneva, 22-26 March 2010. WHO technical report series; n. 949, 2010. p. 7; 36-46.

WORLD HEALTH ORGANIZATION - WHO. Investing to overcome the global impact of neglected tropical diseases: third WHO report on neglected diseases 2015. World Health Organization's Department of Control of Neglected Tropical Diseases. Geneva: WHO Document Production Services, 2015. p. 118-126.

WORLD HEALTH ORGANIZATION - WHO. Leishmaniasis. 2016. Disponível em: <http://www.who.int/tdr/diseases-topics/leishmaniasis/en/>. Acesso em: 25 ago. 2016. 